\title{
Jogo de empresas Simulador de Operações Logísticas - SOLOG: Reação ao seu uso e percepção acerca de sua contribuição para o processo de ensino-aprendizagem de adultos na área de logística
}

\author{
Pítias Teodoro¹, Márcio de Almeida D'Agosto², Yury Vasconcellos da Silva ${ }^{3}$
}

\begin{abstract}
Resumo: O objetivo deste artigo foi o de "Avaliar a adequação do uso do jogo de empresas Simulador de Operações Logísticas - SOLOG - como técnica de apoio ao processo de ensino-aprendizagem de adultos na área de logística". Para tanto, o jogo foi aplicado em alunos de três cursos (Tecnólogo, Graduação e Mestrado) e a análise foi feita a partir de duas abordagens: 1) reação dos participantes ao uso do jogo e 2) percepção da contribuição para o processo de ensinoaprendizagem do uso do jogo. Os resultados mostram que o jogo de empresas SOLOG é adequado ao seu propósito de apoiar o processo de ensino-aprendizagem de adultos na área de logística. Verificou-se que: há oportunidades de melhoria em alguns de seus aspectos, há variáveis com maior influência na reação ao seu uso; há variáveis com maior influência na percepção de sua contribuição para o processo de ensino-aprendizagem e que esses resultados devem ser vistos como um indicativo de aspectos a serem considerados na aplicação de um jogo de empresas, pois, em função das características da pesquisa estes resultados não podem ser generalizados.
\end{abstract}

Palavras-chave: Logística. Jogo de empresas. Ensino-aprendizagem. Andragogia.

\begin{abstract}
This paper aims to "Analyze the adequacy of the use of the business game Logistic Operations Simulator - SOLOG - as a supporting technique to the teaching and learning process for adults in the field of logistics". For doing so, the game was tested with students of three kinds of degrees (Associate, Undergraduate and Master). The analysis was made using two approaches: 1) the reaction of the participants to the game use and 2) the perception of the contribution of the game to the learning process. The results show that the business game SOLOG is suitable to its purpose of supporting the teaching and learning process of adults in the field of logistics. It was observed that: there are opportunities of improvement in some of its aspects, there are variables with a higher influence on the reaction to the use; there are variables with a higher influence on the perception of its contribution to the teaching and learning process and that these results must be seen as an indicator of aspects to be considered when using a business game, for, considering the characteristics of the research, these results cannot be generalized.
\end{abstract}

Keywords: Logistics. Business Game. Teaching and Learning. Andragogy.

\section{INTRODUÇÃO}

O aumento da expectativa de vida da população (CEPAL, 2013; Minayo, 2012; Carvalho e Rodriguez-Wong, 2008) e a redução do ciclo de vida do conhecimento (Lenci, 2010; Grebel. Kraffte Saviott, 2006) são dois dos fenômenos contemporâneos da época atual que podem ser destacados. O primeiro contribui para que o período economicamente ativo das pessoas seja dilatado, enquanto que o segundo leva as pessoas a terem que atualizar suas competências em intervalos cada vez menores. A ocorrência concomitante desses fenômenos acaba por contribuir para um terceiro: "A aprendizagem ao

\footnotetext{
1 Universidade Federal Fluminense e Universidade Federal do Rio de Janeiro (pitiasteodoro@yahoo.com.br).

2 Universidade Federal do Rio de Janeiro (dagosto@pet.coppe.ufr..br).

3 Centro de Ensino Superior - Juiz de Fora - MG

(yury@grupovasconcellos.com.br).
}

Manuscrito recebido em 12/06/2014 e aprovado para publicação em 27/08/2014. Este artigo é parte de TRANSPORTES v. 22, n. 3, 2014. ISSN: 2237-1346 (online).

DOI: http://dx.doi.org/10.14295/transportes.v22i3.803. longo da vida".

A aprendizagem "refere-se às modificações nas capacidades ou disposições do homem que não podem ser atribuídas simplesmente à maturação" (Gil, 2005, p.57). É possível verificar que ocorreu a aprendizagem quando uma pessoa, a partir de suas experiências, adquire competências. Já sua necessidade ao longo da vida é a principal consequência da sociedade do conhecimento (Delors, 2010), fazendo com que não seja mais possível associar a educação apenas às faixas etárias iniciais da vida.

Uma das alternativas para atender à demanda de formação de competências é o uso dos jogos de empresas como apoio ao processo de ensino-aprendizagem. Oliveira (2009), Sauaia (2008), Bernard (2006); Miyashita, Oliveira e Yoshizaki (2003); Martinelli (1987) e Tanabe (1977) defendem que o jogo de empresas é um método educacional que favorece a integração, o repasse e a apreensão dos conteúdos tratados. Verifica-se a aproximação entre teoria e prática sem o tempo, custos e, eventualmente, os riscos 
que a atividade que está sendo simulada poderia proporcionar se o treinamento fosse realizado diretamente no ambiente de trabalho.

Isso é possível porque o ambiente simulado possui um contexto, que exige que os participantes tomem decisões relacionadas aos vários ciclos de gestão simulada (semanas, meses, anos), em um curto espaço de tempo (horas, dias, semanas), sem os ônus (operacionais e ou financeiros) que as possíveis decisões equivocadas provocariam no caso concreto.

Nesse contexto, foi elaborado um jogo de empresas - Simulador de Operações Logísticas - SOLOG, com a finalidade de apoiar o processo de ensino-aprendizagem de adultos na área de logística. Sua primeira versão foi concluída em 2012 e utilizada junto aos alunos de em alguns cursos/disciplinas selecionados ao longo de 2013. São apresentadas e avaliadas a reação ao seu uso e a percepção acerca de sua contribuição para o processo de ensino-aprendizagem. $\mathrm{O}$ uso do jogo de empresas SOLOG ocorreu junto a alunos de turmas dos cursos de Tecnólogo em Logística (SENAI - Rio de Janeiro - RJ), Graduação em Administração (UFF - Volta Redonda RJ) e Mestrado em Administração (UFRRJ -Seropédica - RJ).

A partir do exposto, o presente artigo tem por objetivo "Avaliar a adequação do uso do jogo de empresas Simulador de Operações Logísticas - SOLOG - como técnica de apoio ao processo de ensino-aprendizagem de adultos na área de logística".

A proposição deste trabalho, com foco na área de logística, vai ao encontro de abordagens contemporâneas que tratam da formação de competências em adultos, pois: 1) Considera a necessidade de aprendizagem ao longo da vida (Delors, 2010); 2) Coloca a andragogia como teoria adequada para dar suporte a essa discussão (Knowles, Holton III; Swanson, 2009); 3) Está em consonância com o método da aprendizagem vivencial (Rogers, 1985; Kolb, 1984; Hoover, 1974); 4) Pode ser colocada em prática por meio do emprego dos jogos de empresas como técnica de apoio ao processo de ensino-aprendizagem (Hofstede et al, 2010; Sauaia, 2008; Lewis e Maylor, 2007; Bernard, 2006, Tanabe, 1977) e 5) É direcionado a uma área que é estratégica na gestão das empresas: a logística (Chopra e Meindl, 2011; Ballou, 2007; Bowersox e Closs, 2001).
Quanto à estrutura, o artigo apresenta cinco seções, além dessa introdução e das referências bibliográficas. $\mathrm{Na}$ seção 2 há a revisão bibliográfica sobre a aprendizagem ao longo da vida; na seção 3 é realizada a apresentação do jogo de empresas SOLOG; na seção 4 são definidos os procedimentos metodológicos enquanto que a seção 5 apresenta a discussão e resultados da pesquisa, finalizando com a seção 6 , onde são apresentadas as considerações finais com a síntese dos resultados, limitações e sugestão de estudos complementares.

\section{APRENDIZAGEM AO LONGO DA VIDA}

A aprendizagem ao longo da vida abrange "toda atividade de aprendizagem, em qualquer momento da vida, com o objetivo de melhorar as aptidões, as competências, no quadro de uma perspectiva pessoal, cívica, social e ou relacionada com o emprego" (Sitoe, 2006, p. 284) e que considera, "por um lado, a dimensão temporal da aprendizagem (lifelong) $e$, por outro, a multiplicidade de espaços e contextos de aprendizagem (lifewide). Assim, os indivíduos encontram-se no centro deste processo, onde é a capacidade de construir e de utilizar o conhecimento que é valorizada" (Serapicos, 2008, p.25).

Além da necessidade de novas respostas e com maior frequência, o fator econômico pressiona para que esforços sejam direcionados ao aumento da escolaridade das pessoas também na idade adulta. Pelo relatório da Comissão Internacional sobre Educação para o Século XXI, da Sexta Conferência Internacional de Educação de Adultos, "estima-se hoje que, para cada ano que a média de educação da população adulta aumenta, há um acréscimo correspondente de $3,7 \%$ no crescimento de longo prazo da economia e um acréscimo de $6 \%$ na renda per capita" (MEC, 2010, p. 17).

O reconhecimento da necessidade da aprendizagem, como também sua ocorrência ao longo da vida, coloca o processo de ensinoaprendizagem de adultos em evidência. Em um continuum, que vai da abordagem teórica à alternativa técnica para sua operacionalização, pode-se apresentar a seguinte proposição para a sustentação teórico-prática dessa discussão, nesse trabalho: 1) Abordagem teórica: Andrago- 
gia; 2) Método educacional: Aprendizagem vivencial e 3) Técnica (Estratégia): Jogo de em presas. A Figura 1 sintetiza esta proposição.

Jogo de empresas

Aprendizagem

vivencial

Método educacional

Técnica (Estratégia)

Figura 1 - Sustentação teórica e prática à aprendizagem ao longo da vida Fonte: Elaborado pelos autores, 2014.

A Andragogia é a abordagem teórica que define a aprendizagem de adultos como "a arte $e$ a ciência de orientar adultos a aprender" (Knowles, Holton III; Swanson, 2009, p.65); a Aprendizagem vivencial é um método educacional no qual o aluno é a referência do processo de aprendizagem e o professor atua, predominantemente, como um facilitador (Rogers, 1985); e os Jogos de empresas proporcionam uma vivência dinamizada por um simulador que modela um cenário econômico para a educação profissional (Oliveira, 2009).

Quanto ao reconhecimento do momento em que o aprendiz atinge a idade adulta, condição na qual um processo de ensino-aprendizagem baseado na andragogia deveria ser mais valorizado, não é possível colocar um marco universal e definitivo para todos os indivíduos. Algumas características e ou comportamentos indicam essa situação que, considerando o curso normal da vida, trata-se da pessoa que amadurece biologicamente, assume responsabilidades por meio de decisões próprias e começa a desempenhar papéis socialmente reconhecidos de adultos (Knowles, Holton III; Swanson, 2009).

Há alinhamento entre a abordagem andra- gógica e a aprendizagem vivencial. Enquanto o método da aprendizagem vivencial considera, na situação de aprendizagem, a experiência e a vivência dos alunos (Keys e Biggs, 1990), a abordagem teórica andragógica define que alguns pressupostos devem ser considerados acerca do aprendiz adulto: Auto conceito do aprendiz; Necessidade de saber; Papel da experiência; Prontidão para aprender; Orientação para aprendizagem e Motivação (Knowles, Holton III; Swanson, 2009).

Pode-se dizer que os pressupostos apresentados [na Andragogia] existem em função da experiência e ou vivência dos alunos [destacados na Aprendizagem Vivencial]. Nesta orientação, adota-se uma postura na qual o processo de aprendizagem é ativo (Hoover, 1974).

Embora existam várias abordagens teóricas tratando da aprendizagem, Gil (2005) destaca alguns princípios comuns à maioria das abordagens modernas. Estes aspectos, tratados como princípios educacionais comuns às modernas abordagens sobre aprendizagem, são apresentados na Tabela 1 e relacionados às ações que devem ser realizadas para que o processo de aprendizagem seja favorecido.

Tabela 1 - Princípios educacionais das modernas abordagens sobre aprendizagem

\begin{tabular}{ll}
\hline $\begin{array}{l}\text { Princípios comuns às modernas abordagens sobre } \\
\text { aprendizagem }\end{array}$ & $\begin{array}{l}\text { Ações que podem/devem ser realizadas para favorecer a } \\
\text { aprendizagem }\end{array}$ \\
\hline Diferenças individuais & $\begin{array}{l}\text { Reconhecer as diferenças individuais } \\
\text { Motivação }\end{array}$ \\
Concentração & Manter os alunos atentos \\
Reação & Estimular reações nos alunos \\
Realimentação & Fornecer feedback \\
Memorização & Favorecer a retenção do conhecimento \\
Transferência & Criar condições para que o conhecimento adquirido seja apli- \\
Fonte: Adaptado de Gil, 2005. & cado em outras situações \\
\hline
\end{tabular}

Há alinhamento entre o método da aprendizagem vivencial e a técnica jogo de empresas. Isso ocorre na medida em que o jogo de empresas, por meio de simulação, proporciona aos participantes vivenciarem situações correlatas àquelas que ocorrem no setor e ou empresa que está sendo simulado.

Hofstede et al (2010) reforçam a importância do uso de jogos de empresas no processo de ensino-aprendizagem ao afirmarem que, em 
uma época de crescente especialização em ciências, os jogos de empresas apresentam-se como um outlier, onde o participante se coloca e desempenha o papel de um generalista, não de especialista. Os autores afirmam que os jogos de empresas possibilitam: 1) Integração da cognição, emoção e ação; 2) Forte reprodução da vida social; 3) Multiplicidade de regras e 4) Aprendizagem sobre a vida organizacional e mudança.

\section{JOGO DE EMPRESAS SIMULADOR DE OPERAÇÕES LOGÍSTICAS - SOLOG}

A base da operação de um jogo de empresas é o simulador, também tratado como processador. Trata-se de um instrumento didático constituído "por um conjunto de regras econômicas a serem praticadas para exercitar teorias, conceitos e técnicas" (Sauaia, 2008, p.3). Ainda segundo o autor, o simulador "tem por finalidade propiciar a tomada de decisão e em seguida o exame dos resultados produzidos, dadas as condições iniciais das variáveis do simulador $e$ as relações de causa e efeito sob teste, apoiando o jogo de empresas".

\subsection{Objetivo do jogo de empresas SOLOG}

O Simulador de Operações Logísticas SOLOG - é um software educacional elaborado para apoiar o processo de ensino-aprendizagem na área de logística. O SOLOG opera de maneira interativa - as ações de um dos participantes in- terferem nos resultados dos demais - logo, a incerteza está presente, fazendo com que a simulação ganhe o caráter de jogo [de empresas] (Sauaia, 2008).

A abordagem conceitual do jogo de empresas SOLOG contempla as principais funções da logística que necessitam ser gerenciadas ao longo da cadeia de suprimentos. Conforme Ballou (2007), as funções logísticas primárias são as responsáveis pelo deslocamento e controle de produtos e ou serviços e estão presentes em todos os sistemas logísticos, já as funções logísticas de apoio, dão suporte ao desempenho das atividades primárias e nem todo sistema logístico apresenta todas essas funções.

As funções primárias são: 1) Processamento de pedidos; 2) Manutenção de estoques e 3) Transporte. As funções de apoio são: 1) Armazenagem; 2) Embalagem de produtos; 3) Manuseio de materiais; 4) Planejamento; 5) Sistema de informação e 6) Suprimentos (Chopra e Meindl, 2011; Ballou, 2007; Pozo, 2004; Arnold, 1999).

\subsection{Características do SOLOG}

O jogo de empresas SOLOG é composto por dois módulos: 1) Equipe: para os alunos acessarem as informações do ambiente simulado e registro das decisões e 2) Processamento: para o responsável pela aplicação processar as decisões das equipes e gerar os relatórios gerenciais (parciais e final). A Figura 2 apresenta sua tela principal.

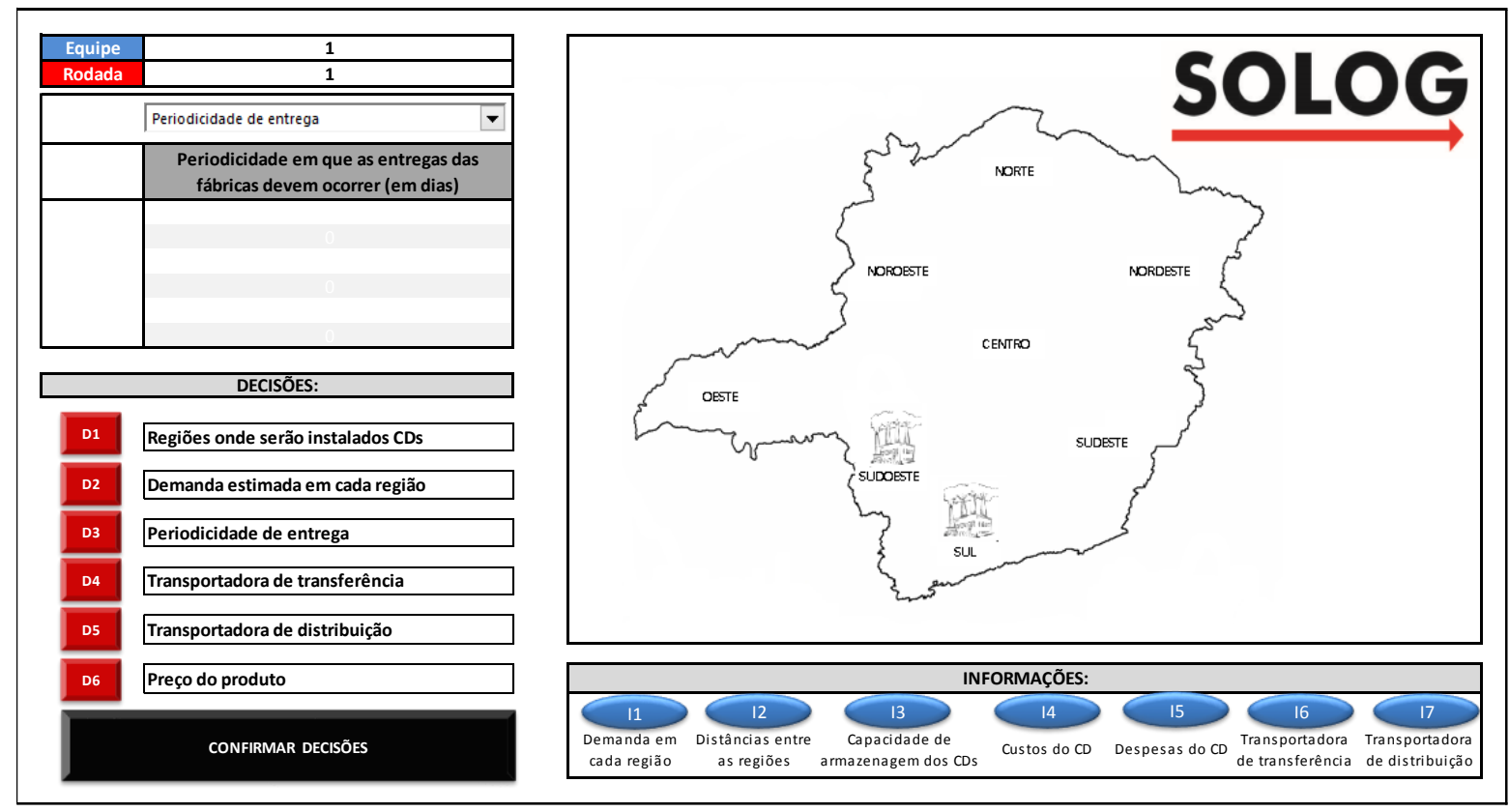

Figura 2 - Tela principal do jogo de empresas SOLOG Fonte: Elaborado pelos autores, 2014 
O ambiente simulado pelo jogo de empresas SOLOG possui oito regiões. Em duas dessas estão localizadas apenas as fábricas (Sudoeste e $\mathrm{Sul}$ ), enquanto que as outras seis regiões (Norte, Noroeste, Nordeste, Centro, Oeste e Sudeste) formam o mercado consumidor. São nessas seis regiões que as empresas que atuam no ambiente do jogo de empresas SOLOG irão instalar seus centros de distribuição (CD), a partir dos quais a fração de demanda conquistada a cada ciclo gerencial será distribuída para os clientes. Para tanto, cada uma das regiões pode receber até um $\mathrm{CD}$ de cada uma das empresas simuladas.

A operação logística simulada envolve a aquisição, a transferência, a armazenagem e a distribuição de um único produto com peso unitário de $1 \mathrm{Kg}$. Para que o produto saia das fábricas e chegue ao cliente são necessárias seis decisões em cada ciclo gerencial. Estas decisões, a principal variável de referência a qual estão vinculadas e as informações disponibilizadas aos participantes são apresentadas na Tabela 2 .

Tabela 2 - Decisões dos gestores e informações fornecidas do ambiente do SOLOG

\begin{tabular}{|c|c|c|}
\hline Decisão & Variável de referência & Informações disponíveis \\
\hline $\begin{array}{l}\text { Volume de produtos } \\
\text { ofertado em cada re- } \\
\text { gião }\end{array}$ & Demanda & Demanda diária de cada uma das seis regiões \\
\hline $\begin{array}{l}\text { Regiões onde serão } \\
\text { instalados os Cen- } \\
\text { tros de Distribuição }\end{array}$ & Localização & $\begin{array}{l}\text { Distância das duas fábricas para as seis regiões (clientes e } \\
\text { CDs) } \\
\text { Distância de cada região para os clientes localizados nas de- } \\
\text { mais regiões } \\
\text { Distância da região para os clientes localizados dentro de sua } \\
\text { própria região }\end{array}$ \\
\hline $\begin{array}{l}\text { Transportadora que } \\
\text { irá realizar a transfe- } \\
\text { rência }\end{array}$ & $\begin{array}{l}\text { Transporte de } \\
\text { Transferência }\end{array}$ & $\begin{array}{l}\text { Nível de serviço (índice de falhas) de cada transportadora de } \\
\text { transferência } \\
\text { Custo do transporte de transferência de cada empresa de } \\
\text { transporte }\end{array}$ \\
\hline $\begin{array}{l}\text { Intervalo entre as } \\
\text { entregas dos produ- } \\
\text { tos ofertados das fá- } \\
\text { bricas para os CDs }\end{array}$ & $\begin{array}{l}\text { Estoque/Lote de } \\
\text { compra }\end{array}$ & Capacidade de armazenagem do CD de cada região \\
\hline $\begin{array}{l}\text { Transportadora que } \\
\text { irá realizar a distri- } \\
\text { buição }\end{array}$ & $\begin{array}{l}\text { Transporte de } \\
\text { Distribuição }\end{array}$ & $\begin{array}{l}\text { Nível de serviço (índice de falhas) de cada transportadora de } \\
\text { distribuição } \\
\text { Custo com transporte de distribuição de cada empresa de } \\
\text { transporte }\end{array}$ \\
\hline \multirow{2}{*}{$\begin{array}{l}\text { Preço de venda para } \\
\text { o produto }\end{array}$} & Despesas dos CDs & $\begin{array}{l}\text { Despesa com aluguel do CD de cada região } \\
\text { Despesa com aluguel extra com CD de cada região } \\
\text { Despesas financeiras referentes ao capital imobilizado com } \\
\text { os estoques }\end{array}$ \\
\hline & Custos dos CDs & $\begin{array}{l}\text { Custo unitário dos produtos } \\
\text { Custos de movimentação de produtos do CD de cada região } \\
\text { Custos de processamento de pedidos de produtos do CD de } \\
\text { cada região }\end{array}$ \\
\hline
\end{tabular}

Fonte: Elaborado pelos autores, 2014

\subsection{Uso do SOLOG}

O jogo de empresas SOLOG simula até 12 ciclos mensais de gestão, com até 30 participantes distribuídos em até 6 empresas. A Tabela 3 detalha em atividades as etapas que devem ser realizadas em seu uso. A etapa de preparação é realizada pelo responsável pela aplicação, as demais, em conjunto com os participantes.

1) Preparação da aplicação do jogo de empresas SOLOG: o interessado em usar o jogo de empresas SOLOG verifica se suas característi- cas possibilitam chegar ao objetivo esperado. A aprendizagem do uso do jogo de empresas SOLOG se dá pela participação em oficinas de formação de multiplicadores e pelo estudo do Manual de Instruções.

2) Aplicação do jogo de empresas SOLOG: esta etapa é subdivida duas: 1) Apresentação do jogo de empresas e 2) Gestão simulada. Na primeira, são discutidas as principais características (conceitos e regras) do ambi- 
ente simulado. Na segunda, os participantes passam a interagir diretamente com o ambiente simulado, assumindo a gestão de uma empresa. Antes de iniciar o jogo propriamente dito, é realizado um jogo teste com apenas um ciclo de gestão de 30 dias.

3) Avaliação da aplicação do jogo de empresas SOLOG: ocorre ao final de cada ciclo ge- rencial e ao final do jogo. Ao final de cada ciclo gerencial os gestores das empresas simuladas recebem um relatório com seu desempenho operacional e financeiro. Estes resultados parciais são consolidados e, ao final do último ciclo gerencial do jogo, a empresa que tiver o maior Lucro líquido (ajustado pela sobra de estoque) é a vencedora.

Tabela 3 - Etapas para aplicação do jogo de empresas SOLOG

\begin{tabular}{|c|c|c|c|}
\hline \multicolumn{2}{|l|}{ Etapas } & \multicolumn{2}{|c|}{ Atividades } \\
\hline \multirow{5}{*}{$\begin{array}{l}\text { Preparação da aplica- } \\
\text { ção do jogo de em- } \\
\text { presas SOLOG }\end{array}$} & \multirow{5}{*}{1 - Preparação } & 1.1 & Verificação da adequação do uso do SOLOG \\
\hline & & 1.2 & Definição da forma de uso do SOLOG \\
\hline & & 1.3 & Definição da forma de avaliação do SOLOG \\
\hline & & 1.4 & Treinamento do(s) mediador(es) \\
\hline & & 1.5 & Manual de Instruções para os alunos \\
\hline \multirow{11}{*}{$\begin{array}{l}\text { Aplicação do jogo de } \\
\text { empresas SOLOG }\end{array}$} & \multirow{7}{*}{$\begin{array}{l}2 \text { - Apresentação do } \\
\text { jogo de empresas } \\
\text { SOLOG }\end{array}$} & 2.1 & Conceito de jogo de empresa \\
\hline & & 2.2 & Objetivo da disciplina/curso \\
\hline & & 2.3 & Objetivo do uso do SOLOG \\
\hline & & 2.4 & Caso simulado do SOLOG \\
\hline & & 2.5 & Esclarecimento sobre as regras do SOLOG \\
\hline & & 2.6 & Definição do número de ciclos gerenciais (rodadas) \\
\hline & & 2.7 & Definição da formação das empresas \\
\hline & \multirow{4}{*}{ 3- Gestão simulada* } & 3.1 & Tomada de decisões \\
\hline & & 3.2 & Registro das decisões \\
\hline & & 3.3 & Processamento das decisões \\
\hline & & 3.4 & Relatório gerencial com resultado do ciclo gerencial \\
\hline \multirow{3}{*}{$\begin{array}{l}\text { Avaliação da aplica- } \\
\text { ção do jogo de em- } \\
\text { presas SOLOG }\end{array}$} & \multirow{3}{*}{ 4-Finalização } & 4.1 & Apresentação do resultado do SOLOG \\
\hline & & 4.2 & Apresentação das opções e resultados das empresas \\
\hline & & 4.3 & Discussão dos resultados do SOLOG (debriefing) \\
\hline
\end{tabular}

\section{PROCEDIMENTOS METODOLÓGICOS}

O presente artigo é de natureza aplicada e possui objetivo descritivo. Quanto aos meios empregados, foi realizada revisão bibliográfica e técnicas padronizadas de coleta de dados (questionário) por meio de levantamento (entrevistas pessoais) (Gil, 2005; Silva e Menezes, 2001).

Para avaliar a reação dos participantes em relação ao uso do jogo de empresas SOLOG adaptou-se o primeiro nível do Modelo de Quatro Etapas de Kirkpatrick e Kirkpatrick (2010a, 2010b) para avaliação de treinamentos. Neste nível do modelo, busca-se "medir a percepção (reação) dos participantes em relação às experiências de aprendizado, ao conteúdo, ao facilitador e à relevância para o trabalho logo após o programa" (Kirkpatrick; Kirkpatrick, 2010a, p.42). Para avaliar a percepção acerca de sua contribuição para o processo de ensino-aprendizagem tomou-se como referência os princípios educacionais das modernas abordagens sobre aprendizagem (Gil, 2005).

A pesquisa foi desenvolvida no nível do indivíduo e a amostra foi selecionada por conveniência (Malhotra, 2001). Os questionários elaborados para mensurar a reação (19 afirmativas) e a contribuição para o processo de ensinoaprendizagem (7 afirmativas) são do tipo estruturado, com respostas em Escala Intervalar de 10 pontos (Mattar, 2008; Malhotra, 2001), além das questões demográficas. A atribuição de um conceito maior às afirmativas (variáveis) indica uma 
reação/percepção mais favorável do respondente considerando as seguintes categorias: Reação/ Percepção Desfavorável: conceitos entre 0 e 5,0; Reação/Percepção Favorável: conceito entre 5,1 e 7,0 e Reação/Percepção Muito favorável: conceito acima de 7,0.A tabulação e tratamento dos dados primários foi realizado por meio do emprego do software Statistical Package for the Social Science (SPSS) que, conforme Hair Jr. et al (1995), tem sido o recurso utilizado no meio acadêmico-científico para produzir estatísticas sobre os atributos da amostra.

\section{RESULTADOS E DISCUSSÃO}

As aplicações da primeira versão do jogo de empresas SOLOG ocorreram no ano de 2013, junto a alunos de cursos/disciplinas selecionados: Tecnólogo em Logística (SENAI - Rio de
Janeiro), Graduação em Administração (UFF Volta Redonda) e Mestrado em Administração (UFRRJ - Seropédica). Participaram, 23 (6 equipes), 30 (6 equipes) e 19 (5 equipes) alunos, dos quais, 8,23 e 18, respectivamente, responderam aos questionários de avaliação. Houve predominância de respondentes do sexo feminino (27 entrevistados) e a idade média do grupo é de 29 anos, variando entre 22 e 50 anos.

\subsection{Reação dos participantes ao uso do jogo de empresas SOLOG}

A Tabela 4 apresenta as médias e os respectivos desvios dos conceitos atribuídos às afirmativas que tratam da reação ao uso do jogo de empresas SOLOG, consolidadas por curso ao qual o respondente é vinculado (Tecnólogo, Graduação e Mestrado) e como um único grupo (Geral).

Tabela 4 - Médias dos conceitos atribuídos à reação ao uso do jogo de empresas SOLOG (continua)

\begin{tabular}{|c|c|c|c|c|c|c|c|c|c|}
\hline & \multirow{2}{*}{ Afirmativas } & \multicolumn{2}{|c|}{ Tecnólogo } & \multicolumn{2}{|c|}{ Graduação } & \multicolumn{2}{|c|}{ Mestrado } & \multicolumn{2}{|c|}{ Geral } \\
\hline & & $\bar{X}$ & $\mathrm{~S}$ & $\bar{X}$ & $\mathrm{~S}$ & $\bar{X}$ & $S$ & $\bar{X}$ & $S$ \\
\hline $\mathrm{p} 1$ & $\begin{array}{c}\text { De uma forma geral o uso do JE SOLOG é adequado para apoiar } \\
\text { processo de aprendizagem }\end{array}$ & 9,0 & 0,89 & 6,6 & 1,76 & 8,1 & 1,47 & 7,5 & 1,82 \\
\hline $\mathrm{p} 2$ & O professor que utilizou o JE SOLOG possui pleno domínio & 9,8 & 0,46 & 8,4 & 2,35 & 9,3 & 0,56 & 8,9 & 1,79 \\
\hline p3 & Reconheço o que é um jogo de empresas & 7,5 & 1,60 & 8,9 & 1,22 & 9,1 & 0,81 & 8,7 & 1,31 \\
\hline $\mathrm{p} 4$ & $\begin{array}{c}\text { Sei qual o objetivo da disciplina na qual o jogo de empresas foi } \\
\text { usado }\end{array}$ & 7,2 & 3,26 & 7,5 & 2,08 & 8,9 & 0,94 & 7,9 & 2,11 \\
\hline p5 & Sei qual o objetivo do uso de um jogo de empresas & 7,7 & 1,49 & 8,4 & 1,72 & 9,3 & 0,56 & 8,6 & 1,50 \\
\hline p6 & Compreendi o caso simulado pelo JE SOLOG & 8,1 & 1,55 & 7,4 & 1,63 & 8,4 & 1,19 & 7,8 & 1,55 \\
\hline $\mathrm{p} 7$ & Entendi as regras do JE SOLOG & 8,4 & 1,69 & 6,9 & 2,30 & 8,4 & 1,44 & 7,6 & 2,08 \\
\hline $\mathrm{p} 8$ & Sei como são formadas as equipes para o JE SOLOG & 7,4 & 3,29 & 7,9 & 3,35 & 8,9 & 1,76 & 8,1 & 2,93 \\
\hline $\mathrm{p} 9$ & $\begin{array}{c}\text { A rodada teste tirou todas as dúvidas que ainda tinha sobre o JE } \\
\text { SOLOG }\end{array}$ & 6,1 & 2,80 & 4,1 & 2,53 & 6,3 & 3,33 & 5,1 & 3,02 \\
\hline p10 & $\begin{array}{c}\text { As informações para as tomadas de decisão no ambiente simulado } \\
\text { do JE SOLOG são claras }\end{array}$ & 7,3 & 1,83 & 6,1 & 2,14 & 7,0 & 1,42 & 6,6 & 1,93 \\
\hline p11 & $\begin{array}{l}\text { A planilha/formulário aonde as decisões foram registradas é ade- } \\
\text { quada }\end{array}$ & 8,4 & 1,19 & 7,2 & 2,16 & 8,3 & 1,51 & 7,8 & 1,90 \\
\hline p12 & $\begin{array}{l}\text { O tempo para processar e gerar o relatório parcial não atrapalhou a } \\
\text { aplicação do JE SOLOG }\end{array}$ & 6,8 & 1,91 & 8,1 & 2,15 & 8,0 & 2,69 & 7,8 & 2,33 \\
\hline p13 & As informações apresentadas no relatório parcial são claras & 7,6 & 1,85 & 7,6 & 2,13 & 8,5 & 1,44 & 7,9 & 1,90 \\
\hline p14 & As informações apresentadas no relatório parcial são suficientes & 6,5 & 3,07 & 6,8 & 1,97 & 6,7 & 1,73 & 6,7 & 2,10 \\
\hline p15 & $\begin{array}{l}\text { A forma como as informações foram apresentadas no relatório par- } \\
\text { cial é adequada }\end{array}$ & 7,6 & 1,51 & 7,4 & 1,86 & 7,9 & 1,47 & 7,6 & 1,68 \\
\hline p16 & $\begin{array}{l}\text { A forma como é feita a avaliação dos resultados das equipes do JE } \\
\text { SOLOG é clara }\end{array}$ & 8,0 & 1,07 & 6,9 & 1,79 & 7,2 & 1,81 & 7,2 & 1,73 \\
\hline
\end{tabular}


Afirmativas

$\begin{array}{llllllll}\bar{X} & \mathrm{~S} & \bar{X} & \mathrm{~S} & \bar{X} & \mathrm{~S} & \bar{X} & \mathrm{~S}\end{array}$

p17 A discussão sobre o desempenho das equipes tirou todas as minhas dúvidas de como os resultados foram avaliados

\begin{tabular}{|l|l|l|l|l|l|l|l|}
6,3 & 3,24 & 6,1 & 2,06 & 7,6 & 1,98 & 6,6 & 2,35 \\
\hline 7,1 & 3,44 & 7,1 & 2,48 & 7,5 & 2,53 & 7,2 & 2,65 \\
\hline
\end{tabular}

p18 Concordo com o resultado obtido no JE SOLOG pela minha equipe $\quad 7,1 \quad 3,44 \quad 7,1 \quad 2,48 \quad 7,5 \quad 2,53 \quad 7,2 \quad 2,65$

p19 Fiquei satisfeito com o resultado obtido no JE SOLOG pela minha equipe

$\begin{array}{llllllll}8,6 & 1,51 & 5,8 & 3,37 & 6,1 & 3,89 & 6,4 & 3,46\end{array}$

Fonte: Elaborado pelos autores, 2014.

Entre as células que contém as médias $(\bar{X})$ dos conceitos que indicam a reação dos respondentes na Tabela 4, àquelas que estão em cor cinza destacam as afirmativas cuja reação dos respondentes foi Desfavorável (Conceito entre 0 e 5,0) ou Favorável (Conceito entre 5,1 e 7,0), enquanto que as demais apresentam reação
Muito Favorável (Conceito acima de 7,0).

Por meio da Figura 3, é possível visualizar as diferenças de reação dos respondentes a cada um dos itens analisados. Quanto mais próxima do centro estiver a curva, pior é a reação do grupo em relação ao item em análise, quanto mais próxima da borda, melhor.

\section{Média do conceito sobre a reação ao uso do SOLOG}
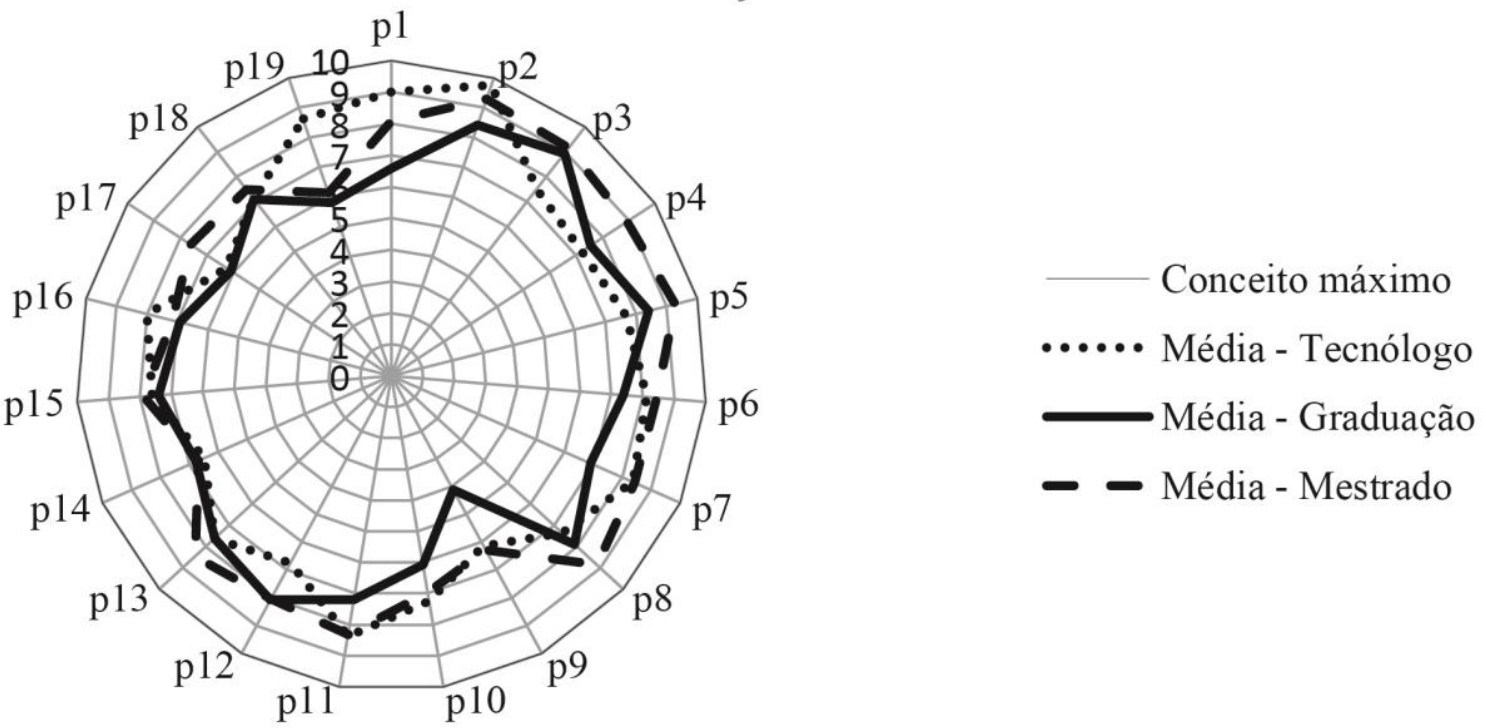

Figura 3 - Média do conceito atribuído à reação ao uso do jogo de empresas SOLOG por curso Fonte: Elaborado pelos autores, 2014.

Entre os 19 itens utilizados para mensurar a adequação do uso do jogo de empresas SOLOG, verificou-se que em 10 itens $(52,6 \%)$ houve reação Muito Favorável (Conceito acima de 7,0) pelos respondentes dos três cursos no qual o jogo foi aplicado; em 5 itens $(26,3, \%)$ houve reação Muito Favorável pelos respondentes de dois dos cursos e em 2 itens (10,5\%) houve reação Muito Favorável por respondentes de um dos cursos.

Os dois itens analisados que foram apontados como Desfavorável (Conceito entre 0 a
5,0) e ou Favorável (Conceito entre 5,1 e 7,0) pelos alunos dos três cursos são: (p9: A rodada teste tirou todas as dúvidas que ainda tinha sobre o JE SOLOG e p14: As informações apresentadas no relatório parcial são suficientes). Dois outros itens foram apontados como Favorável (Conceito entre 5,1 e 7,0) por respondentes de dois cursos: (p17: A discussão sobre o desempenho das equipes tirou todas as minhas dúvidas de como os resultados foram avaliados e p19: Fiquei satisfeito com o resultado obtido no 
JE SOLOG pela minha equipe). A princípio, estes são aspectos que devem ser objeto de maior atenção pelo responsável pela aplicação quando a análise está voltada para a reação ao uso do jogo de empresas SOLOG.

Algumas dúvidas muito simples foram apontadas pelos participantes durante as aplicações do jogo de empresas SOLOG, sugerindo que alguns dos alunos não haviam lido o Manual de Instruções com antecedência. Isso justificaria um conceito mais crítico, e por um número maior de respondentes, às afirmações p9, p14 e p17. Quanto à afirmativa p19, que trata da satisfação em relação ao resultado obtido pela equipe, verificou-se correlação positiva entre a posição da equipe e a opinião do respondente. Quanto pior o resultado da equipe, pior foi o conceito médio do respondente à afirmativa.

Ainda no intuito de verificar a adequação do uso do jogo de empresas SOLOG nos diferentes cursos aos quais foi aplicado (Tecnólogo, Graduação ou Mestrado), foi calculada a média das médias dos conceitos atribuídos às afirmativas relacionadas à reação ao seu uso. A reação dos respondentes dos três cursos pode ser classificada como Muito Favorável (Conceito acima de 7,0), com médias gerais de 8,0 para os alunos de Mestrado, 7,6 para os alunos de Tecnólogo e 7,1 para os alunos de Graduação, colocando o uso do jogo de empresas SOLOG como adequado para os três níveis de cursos nos quais foi utilizado. As médias das médias e os respectivos desvios estão apresentados na Tabela 5.

Tabela 5 - Média das médias do conceito atribuído à reação ao uso do jogo de empresas SOLOG

\begin{tabular}{cccccccccc}
\hline \multirow{2}{*}{ Questões } & \multicolumn{2}{c}{ Tecnólogo } & \multicolumn{2}{c}{ Graduação } & \multicolumn{2}{c}{ Mestrado } & \multicolumn{2}{c}{ Geral } \\
\cline { 2 - 9 } & $\bar{X}$ & $\mathrm{~S}$ & $\bar{X}$ & $\mathrm{~S}$ & $\bar{X}$ & $\mathrm{~S}$ & $\bar{X}$ & $\mathrm{~S}$ \\
\hline Todas (p1 a p19) & 7,6 & 0,93 & 7,1 & 1,11 & 8,0 & 0,98 & 7,6 & 0,91 \\
\hline & \multicolumn{3}{c}{ Fonte: Elaborado pelos autores, 2014.} \\
\end{tabular}

Embora a reação ao uso do jogo de empresas SOLOG tenho o colocado como adequado pelos respondentes de todos os cursos, houve diferença de 0,9 entre a maior e a menor média geral dos cursos. Em termos absolutos trata-se de uma diferença pequena, mas pode-se afirmar que foi significativa (valor- $p=0,038$ ). Por meio do teste de contraste de Tukey (valor-p $=0,030$ ) verificou-se que há diferença significativa entre a reação ao uso do jogo dos alunos dos cursos de Graduação e Mestrado e que não há diferença significativa entre a reação ao seu uso entre os alunos de Tecnólogo e Graduação ou entre os alunos de Tecnólogo e Mestrado.

É possível relacionar os conceitos médios referentes à reação dos respondentes ao uso do jogo de empresas SOLOG com seu nível de conhecimento prévio acerca de jogos de empresas. Entre os participantes do curso de Tecnólogo que responderam ao questionário, um aluno (12,5\% dos respondentes) conhecia a técnica e nunca havia participado de uma aplicação, entre os participantes do curso de mestrado, 4 alunos (22,2\% dos respondentes) conheciam a técnica e os 4 já haviam participado de pelo menos uma aplicação de um jogo de empresas, enquanto que entre os alunos do curso de graduação, 22 alunos (95,7\% dos respondentes) conheciam a técnica e os 22 já haviam participado de pelo menos uma aplicação de um jogo de empresas. Verificou-se que os alunos com maior conhecimento prévio tiveram a reação mais crítica ao uso de jogo de empresas do SOLOG.

Para completar a análise acerca da adequação do uso do jogo de empresas SOLOG, a afirmativa p1: "De uma forma geral o uso do JE SOLOG é adequado para apoiar processo de aprendizagem", foi tratada como aquela que mede, de forma geral, a opinião do entrevistado. A mesma foi utilizada como variável dependente em um modelo de regressão múltipla cujo objetivo foi o de identificar quais as variáveis (afirmativas) que mais explicam a reação ao uso do jogo de empresas SOLOG para apoio ao processo de ensino-aprendizagem de adultos na área de logística, de forma geral.

O modelo de regressão expresso pela Equação 1 foi considerado significativo (valor-p $\sim 0,00$ ) para prever a reação do respondente acerca do uso do jogo de empresas SOLOG, tendo obtido $R^{2}=0,636$, logo, explica $63,6 \%$ da variabilidade de $Y$.

$$
\begin{aligned}
Y= & -0,481+0,257 X_{1}+0,263 X_{2} \\
& +0,313 X_{3}+0,059 X_{4}
\end{aligned}
$$


Onde:

$Y=$ Conceito da afirmativa p1: De uma forma geral o uso do JE SOLOG é adequado para apoiar processo de aprendizagem;

$X_{1}=$ Conceito da afirmativa p10: As informações para as tomadas de decisão no ambiente simulado do JE SOLOG são claras;

$X_{2}=$ Conceito da afirmativa p7: Entendi as regras do JE SOLOG;

$X_{3}=$ Conceito da afirmativa p13: As informações apresentadas no relatório parcial são claras;

$X_{4}=$ Idade do respondente.

A importância de cada variável para a explicação da variável dependente pode ser verificada por meio do $\beta_{\mathrm{p}}$ (Beta padronizado): $\beta_{\mathrm{p} 1}=$ 0,$279 ; \beta_{\mathrm{p} 2}=0,308 ; \beta_{\mathrm{p} 3}=0,320$ e $\beta_{\mathrm{p} 4}=0,248$. Assim, é possível apresentar as variáveis que explicam o modelo em ordem de importância: p13: As informações apresentadas no relatório parcial são claras $\left(\beta_{\mathrm{p} 3}=0,320\right) ; \mathrm{p} 7$ : Entendi as regras do JE SOLOG $\left(\beta_{\mathrm{p} 2}=0,308\right)$; $\mathrm{p} 10$ : As infor- mações para as tomadas de decisão no ambiente simulado do JE SOLOG são $\operatorname{claras}\left(\beta_{\mathrm{p} 1}=\right.$ $0,279)$ e Idade do respondente $\left(\beta_{\mathrm{p} 4}=0,248\right)$.

Ao identificar quais são os aspectos que terão maior influência na reação ao uso do jogo de empresas SOLOG, o interessado em utilizálo como técnica de apoio ao processo de ensinoaprendizagem poderá obter melhores resultados ao considerá-los no planejamento e na aplicação do jogo.

\subsection{Percepção dos participantes acerca da contribuição do uso do jogo de empresas SOLOG para o processo de ensino-aprendizagem}

A Tabela 6 apresenta as médias e os respectivos desvios dos conceitos atribuídos às afirmativas que tratam da percepção acerca da contribuição do uso do jogo de empresas SOLOG para o processo de ensino-aprendizagem, consolidadas por curso ao qual o respondente é vinculado (Tecnólogo, Graduação e Mestrado) e como um único grupo (Geral).

Tabela 6 - Médias dos conceito atribuídos à percepção acerca da contribuição do uso do jogo de empresas SOLOG para o processo de ensino-aprendizagem

\begin{tabular}{|c|c|c|c|c|c|c|c|c|c|}
\hline \multirow{2}{*}{\multicolumn{2}{|c|}{$\begin{array}{c}\text { Afirmativas } \\
\text { Considero que o uso do jogo de empresas criou condi- } \\
\text { ções para que cada participante ... }\end{array}$}} & \multicolumn{2}{|c|}{ Tecnólogo } & \multicolumn{2}{|c|}{ Graduação } & \multicolumn{2}{|c|}{ Mestrado } & \multicolumn{2}{|c|}{ Geral } \\
\hline & & \multirow{2}{*}{$\bar{X}$} & \multirow{2}{*}{ S } & \multirow{2}{*}{$\begin{array}{l}\bar{X} \\
6,0\end{array}$} & \multirow{2}{*}{$\begin{array}{c}S \\
1,84\end{array}$} & \multirow{2}{*}{$\bar{X}$} & \multirow{2}{*}{$\begin{array}{c}S \\
1,46\end{array}$} & \multirow{2}{*}{$\overline{\bar{X}}$} & \multirow{2}{*}{$\begin{array}{c}S \\
1,99\end{array}$} \\
\hline p. 1 & $\begin{array}{c}\text {... tenha o processo de aprendizagem em seu } \\
\text { próprio ritmo }\end{array}$ & & & & & & & & \\
\hline p.2 & $\begin{array}{c}\text {... fique motivado em participar do processo de } \\
\text { aprendizagem }\end{array}$ & 9,0 & 0,83 & 7,0 & 1,83 & 9,0 & 1,38 & 8,0 & 1,81 \\
\hline p.3 & ... fique concentrado no que está aprendendo & 9,0 & 0,76 & 7,0 & 1,72 & 9,0 & 1,45 & 8,0 & 1,76 \\
\hline p.4 & $\begin{array}{l}\text {... se manifeste espontaneamente quanto ao pro- } \\
\text { cesso de aprendizagem }\end{array}$ & 8,5 & 1,30 & 8,0 & 1,53 & 8,0 & 1,53 & 8,0 & 1,52 \\
\hline p.5 & $\begin{array}{l}\text {... reconheça erros e acertos durante o processo } \\
\text { de aprendizagem }\end{array}$ & 9,0 & 0,82 & 8,0 & 1,74 & 8,0 & 1,54 & 8,0 & 1,60 \\
\hline p.6 & ... memorize (retenha) o aprendizado & 9,0 & 1,16 & 8,0 & 1,62 & 9,0 & 1,02 & 9,0 & 1,41 \\
\hline p.7 & ... aplique o que aprendeu em outras situações & 8,5 & 1,30 & 8,0 & 2,04 & 9,0 & 1,30 & 9,0 & 1,75 \\
\hline
\end{tabular}

Entre as células da Tabela 6 que contém as médias $(\bar{X})$ dos conceitos que indicam a percepção acerca da contribuição do uso do jogo de empresas para o processo de ensino-aprendizagem, àquelas que estão em cor cinza destacam os itens aos quais a percepção dos respondentes foi Favorável (Conceito entre 5,1 e 7,0), enquanto que as demais apresentam percepção Muito Favorável (Conceito acima de 7,0). Não houve percepção Desfavorável (Conceito entre 0 e 5,0) a nenhum dos itens avaliados.

Por meio da Figura 4, é possível visualizar as diferenças de percepção dos respondentes a cada um dos itens analisados. Quanto mais próxima do centro estiver a curva, pior é a percepção do grupo em relação ao item em análise, quanto mais próxima da borda, melhor.

Entre os 7 itens utilizadas para mensurar a adequação de seu uso, verificou-se que em 4 itens $(57,1 \%)$ houve percepção Muito Favorável (Conceito acima de 7,0) pelos respondentes dos três cursos no qual o jogo foi aplicado; em 2 itens $(28,6 \%)$ houve percepção Muito Favorável pelos respondentes de dois dos cursos e em 1 item (14,3\%) houve percepção Muito Favorável por respondentes de um dos cursos. 


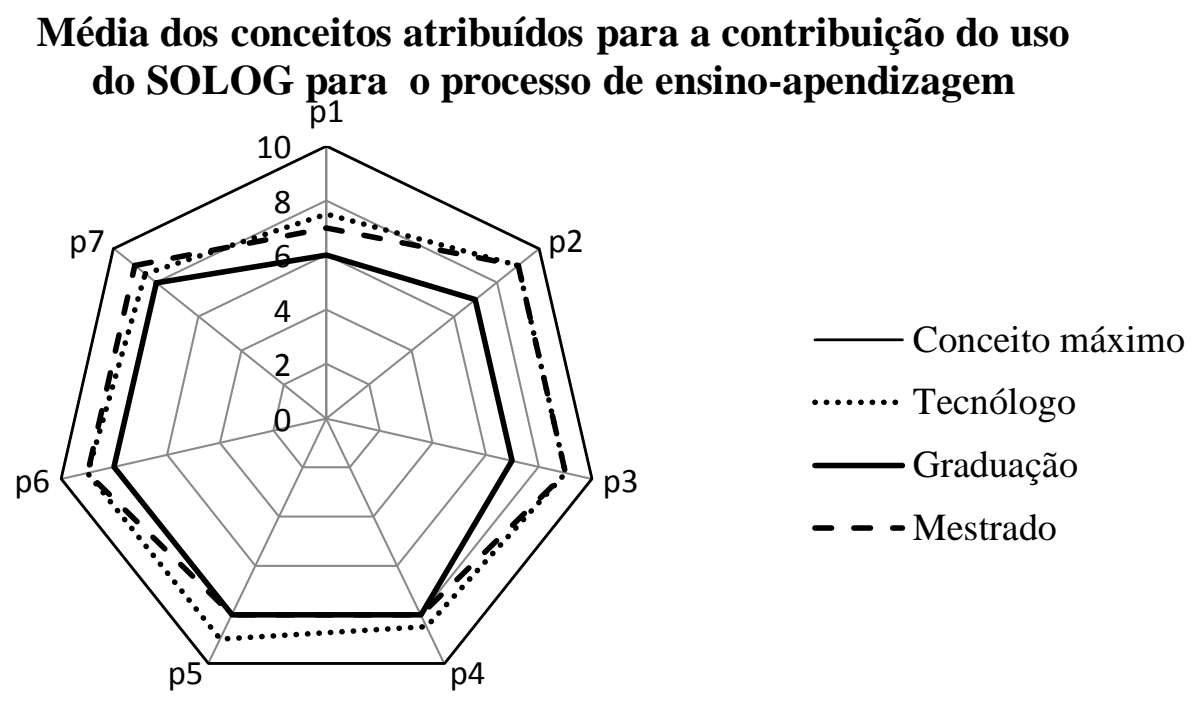

Figura 4 - Média dos conceitos atribuídos à contribuição do uso do SOLOG para o processo de ensino-aprendizagem por curso Fonte: Elaborado pelos autores, 2014.

Verificou-se que um dos itens analisados foi apontado como Favorável (Conceito entre 5,1 e 7,0) pelos alunos dos três cursos (p1: Considero que o uso do jogo de empresas criou condições para que cada participante tenha o processo de aprendizagem em seu próprio ritmo). A princípio, este é o aspecto que deve ser objeto de maior atenção pelo responsável pela aplicação quando a análise está voltada para a percepção acerca da contribuição para o processo de ensino-aprendizagem do uso do jogo de empresas SOLOG.

Ainda no intuito de verificar a adequação do uso do jogo de empresas SOLOG nos dife- rentes cursos aos quais foi aplicado (Tecnólogo, Graduação ou Mestrado), foi calculada a média das médias dos conceitos atribuídos às afirmativas relacionadas à percepção acerca da contribuição de seu uso. A percepção dos respondentes dos três cursos pode ser classificada como Muito Favorável (Conceito acima de 7,0), com médias gerais de 8,5 para os alunos de Tecnólogo, 8,3 para os alunos de Mestrado e 7,2 para os alunos de Graduação, colocando o uso do jogo de empresas SOLOG como adequado para os três níveis de cursos nos quais foi utilizado. As médias das médias e os respectivos desvios estão apresentados na Tabela 7.

Tabela 7 - Média das médias do conceito à contribuição do uso do jogo de empresas SOLOG para o processo de ensino-aprendizagem

\begin{tabular}{lccccccccc}
\hline \multirow{2}{*}{ Questões } & \multicolumn{2}{c}{ Tecnólogo } & \multicolumn{2}{c}{ Graduação } & Mestrado & \multicolumn{2}{c}{ Geral } \\
\cline { 2 - 9 } & $\bar{X}$ & $\mathrm{~S}$ & $\bar{X}$ & $\mathrm{~S}$ & $\bar{X}$ & $\mathrm{~S}$ & $\bar{X}$ & $\mathrm{~S}$ \\
\hline Todas (p1 a p7) & 8,5 & 1,02 & 7,2 & 1,36 & 8,3 & 1,06 & 8,0 & 0,69 \\
\hline Fonte: Elaborado pelos autores, 2014. & & & & & & & &
\end{tabular}

Embora o jogo de empresas SOLOG tenha sido considerado adequado pelos respondentes de todos os cursos, houve diferença de 1,3 entre a maior e a menor média geral dos cursos. Em termos absolutos trata-se de uma diferença pequena, mas pode-se afirmar que foi significativa (valor-p $=0,038$ ). Por meio do teste de contraste de Tukey (valor-p $=0,012$ ) verificou-se que há diferença significativa entre a percepção dos alunos dos cursos de Graduação e Mestrado e que não há diferença significativa entre a reação dos alunos de Tecnólogo e Graduação (vapor $p$ $=0,55$ ) ou de Tecnólogo e Mestrado (valor $\mathrm{p}=$
0,976).

Para completar a análise acerca da adequação do uso do jogo de empresas SOLOG, a afirmativa p1: "De uma forma geral o uso do JE SOLOG é adequado para apoiar processo de aprendizagem”, foi tratada como aquela que mede, de forma geral, a opinião do entrevistado. A mesma foi utilizada como variável dependente em um modelo de regressão múltipla cujo objetivo foi o de identificar quais as variáveis (afirmativas) que mais explicam a percepção acerca da contribuição do uso do jogo de empresas SOLOG para apoio ao processo de ensino- 
aprendizagem de adultos na área de logística, de forma geral.

O modelo de regressão expresso pela Equação 2 foi considerado significativo (valor-p 0,00) para prever a reação do respondente acerca do uso do jogo de empresas SOLOG, tendo obtido $R^{2}=0,492$, logo, que explica $49,2 \%$ da variabilidade de $\mathrm{Y}$.

$\mathrm{Y}=0,57+0,586 \mathrm{X}_{1}+0,074 \mathrm{X}_{2}$

Onde:

$Y=$ Conceito da afirmativa p1: De uma forma geral o uso do JE SOLOG é adequado para apoiar processo de aprendizagem;

$X_{1}=$ Conceito da afirmativa p7: Considero que o uso do jogo de empresas SOLOG criou condições para que cada participante aplique o que aprendeu em outras situações;

$X_{2}=$ Idade do respondente.

A importância de cada variável para a explicação da variável dependente pode ser verificada por meio do $\beta_{\mathrm{p}}$ (Beta padronizado): $\beta_{\mathrm{p} 1}=$ 0,$577 ; \beta_{\mathrm{p} 2}=0,287$. Assim, é possível apresentar as variáveis que explicam o modelo em ordem de importância: p18: As informações apresentadas no relatório parcial são claras $\left(\beta_{\mathrm{p} 1}=0,577\right)$ e Idade do respondente $\left(\beta_{\mathrm{p} 2}=0,287\right)$.

Ao identificar quais são os aspectos que terão maior influência na percepção acerca da contribuição do uso do jogo de empresas SOLOG para o processo de ensino-aprendizagem, o interessado em utilizá-lo poderá obter melhores resultados ao considerá-los no planejamento e na aplicação do jogo.

\section{CONSIDERAÇÕES FINAIS}

A partir do objetivo deste trabalho, que foi o de "Avaliar a adequação do uso do jogo de empresas Simulador de Operações Logísticas - SOLOG - como técnica de apoio ao processo de ensino-aprendizagem de adultos na área de logística", concluiu-se que este jogo de empresas é adequado para seu propósito. Tanto na avaliação da reação ao seu uso, quanto na avaliação da percepção acerca de sua contribuição para o processo de ensino-aprendizagem, as médias gerais dos conceitos atribuídos aos itens de análise, em maioria absoluta, foi acima de 7,0, apresentando conceito Muito Favorável.

$\mathrm{Na}$ avaliação da reação ao uso, verificou- se que os aspectos tratados em 7 das 19 afirmativas, mais uma característica demográfica (Idade), devem ser consideradas de forma diferenciada no uso do jogo de empresas SOLOG. Quatro dessas afirmativas devem ser tratadas como oportunidades de melhorias e as outras quatro como os fatores que influenciam mais fortemente uma reação mais favorável ao uso do jogo.

Os itens analisados que devem ser aprimorados estão concentrados nas etapas de Gestão simulada e Apresentação dos resultados. Na etapa da Gestão simulada, é necessário investigar a contribuição da rodada-teste para tirar as dúvidas sobre o jogo de empresas SOLOG e se o volume de informações apresentadas no relatório gerencial está adequado (p9: A rodada teste tirou todas as dúvidas que ainda tinha sobre o JE SOLOG e p14: As informações apresentadas no relatório parcial são suficientes).

Em relação à Apresentação dos resultados, a forma como a discussão sobre o desempenho e resultados das empresas está sendo conduzida, bem como o nível de satisfação em relação ao próprio resultado, são os itens a serem investigados (p17: A discussão sobre o desempenho das equipes tirou todas as minhas dúvidas de como os resultados foram avaliados e p19: Fiquei satisfeito com o resultado obtido no JE SOLOG pela minha equipe). As melhorias podem estar associadas ao uso do jogo de empresas SOLOG, ao conteúdo do Manual de Instruções e ou ao próprio simulador.

Por outro lado, o modelo de regressão apontou para quatro outros aspectos que respondem, em conjunto, por $63,6 \%$ da reação dos participantes. As variáveis que explicam o modelo em ordem de importância são: p13: As informações apresentadas no relatório parcial são claras; p7: Entendi as regras do JE SOLOG; p10: As informações para as tomadas de decisão no ambiente simulado do JE SOLOG são claras e Idade dos respondentes, logo, devem ser considerados de forma diferenciada no uso do jogo de empresas SOLOG. Destaca-se, ainda, que nenhuma das variáveis do modelo está entre àquelas que obtiveram os conceitos mais baixos por alunos de dois ou três cursos.

$\mathrm{Na}$ avaliação da percepção acerca da contribuição do uso do jogo de empresas para a aprendizagem, verificou-se que os aspectos tratados em 2 das 7 afirmativas, mais uma característica demográfica (Idade), devem ser consi- 
deradas de forma diferenciada no uso do jogo de empresas SOLOG. Uma dessas afirmativas deve ser tratada como oportunidade de melhoria e as outras duas como os fatores que influenciam mais fortemente uma percepção mais favorável ao uso do jogo.

A afirmativa que deve ser tratada como oportunidade de melhoria é a p7: Considero que o uso do jogo de empresas SOLOG criou condições para que cada participante tenha o processo de aprendizagem em seu próprio ritmo. As várias atividades do jogo (síncronas e assíncronas) deveriam contribuir para que cada participante pudesse ditar seu ritmo de aprendizagem, entretanto, as respostas dos entrevistados contrariam isso.

Por outro lado, o modelo de regressão apontou para dois outros aspectos que respondem, em conjunto, por 49,2\% da percepção dos participantes em relação à contribuição do jogo de empresas para o processo de aprendizagem. As variáveis que explicam o modelo em ordem de importância são: p7: Considero que o uso do jogo de empresas SOLOG criou condições para que cada participante aplique o que prendeu em outras situações e Idade dos respondentes, logo, devem ser considerados de forma diferenciada no uso do jogo de empresas SOLOG. Destacase, ainda, que nenhuma das variáveis do modelo está entre àquelas que obtiveram os conceitos mais baixos por alunos de dois ou três cursos.

Quanto às limitações do trabalho, os questionários desenvolvidos para avaliar reação e percepção quanto à contribuição para a aprendizagem não foram validados. Durante as análises foi identificada a necessidade de inserir uma afirmativa referente à leitura prévia do Manual de Instruções, pois isso permitiria verificar se os itens que foram criticados realmente têm que ser ajustados. Os resultados ora apresentados devem ser considerados para o grupo de alunos que participou das aplicações e respondeu à pesquisa. Ainda em relação aos resultados, as conclusões comparativas realizadas a partir das respostas dos alunos do curso de tecnólogo podem estar enviesadas uma vez que foi baixo número de respostas obtidas junto aos participantes deste curso. Embora o resultado apurado seja um indicativo positivo quanto à adequação do uso do SOLOG como técnica de apoio ao processo de ensino-aprendizagem de adultos na área de logística, é necessário realizar novos estudos para que seja possível generalizar essa afirmação.
Uma oportunidade é a avaliação do jogo de empresas SOLOG a partir do $2^{\circ}$ nível do modelo de Quatro Etapas: Aprendizagem.

Ainda assim, é possível considerar os aspectos identificados na aplicação do jogo de empresas SOLOG, em aplicações, inclusive, de outros jogos de empresas. Assim, seria possível programar a aplicação obtendo os melhores resultados no processo de ensino-aprendizagem, pois, há a possibilidade de reduzir interferências e, ao mesmo tempo, reforçar as potencialidades.

\section{REFERÊNCIAS}

Arnold, J. R. T. (1999) Administração de materiais. Administração de materiais: uma introdução. São Paulo: Atlas, 1999.

Ballou, R. H. (2007). Logística Empresarial. São Paulo: Atlas.

Bernard, R. (2006). Métodos e jogos de empresas/simulação gerencial. In: Marion, A. L. C, Marion, J. C. Marion, Metodologias de Ensino na Área de Negócios. São Paulo: Atlas.

Bowersox, D. J. Closs, D. J. (2001) Logística empresarial: o processo de integração da cadeia de suprimento. São Paulo: Atlas.

Carvalho, J. A. M. de; Rodriguez-Wong, L. L. (2008) A transição da estrutura etária da população brasileira na primeira metade do século XXI. Cad. Saúde Pública, Rio de Janeiro, v. 24, n.3, Mar. 2008. Available from http://www.scielo.br/scielo.php?script=sci_arttext\&pid=S0102$311 X 2008000300013 \& \operatorname{lng}=$ en\&nrm=iso Access on 28 Jan. 2014. DOI: 10.1590/S0102-311X2008000300013

CEPAL (2013) Comissão Econômica para a América Latina e o Caribe. Panorama Social da América Latina. Nações Unidas CEPAL, 2013. Disponível em www.cepal.org.publicaciones/ xlm/7/51767/PanoramaSocialDA2013DocInf.pdf . Acesso em 28 de janeiro de 2014.

Chopra, S.; Meindl, P. (2011) Gestão da cadeia de suprimento: estratégia, planejamento e operações. São Paulo: Pearson Prentice Hall.

Delors, J. (2010). Educação: um tesouro a descobrir. Relatório para a UNESCO da Comissão Internacional para a Educação no Século XXI. Brasília, julho de 2010.

Gil, A.C. (2005) Metodologia do ensino superior. São Paulo: Atlas.

Grebel, T.; Krafft, J.; Saviotto, P. P. (2006) On the Life Cycle of Knowledge Intensive Sectors.Revue de l'OFCE 5/2006 (n ${ }^{\circ} 97$ bis), p.63-85.URL: www.cairn.info/revue-de-1-ofce-2006-5page-63.htm. DOI: 10.3917/reof.073.85. Acesso em 20 de janeiro de 2014.

Hair Jr., J. F.; Anderson, R. E.; Tathan, R. L.; Black, W. C. (1995) Multivariate das analysis: whith readings. New Jersey: Pretince Hall.

Hofstede, G. J.; Caluwé, L.de; Peters, V. (2010) Synthesis Why Simulation Games Work-In Search of the Active Substance: A Synthesis. Simulation Gaming 2010 41: 824 originally published online 5 August 2010. DOI: 10.1177/1046878110375596. Disponível em: http://sag.sagepub.com/content/41/6/824 
Hoover, J. D (1974) Experiential learning: conceptualization and definition. In: Kenderdin, J.; Keys, B. (Eds.). Simulation, games and experiential learning techniques: on the road to a new frontier, 1., 1974, Oklahoma. Anais... Oklahoma: ABSEL, 1974. p. 31-35. CD-ROM.

Keys, J. B; Biggs, W. D.(1990) A review of business games. In: Gentry, J. W. Guide to Business Gaming and Experimental Learning. London: Nichols G P Publishing, 1990. p. 48-73.

Kirkpatrick, D. L; Kirkpatrick, J. D. (2010a) Como avaliar programas de treinamento de equipes: os quarto níveis. Rio de Janeiro: SENAC Rio.

Kirkpatrick, D..L; Kirkpatrick, J. D. (2010b) Como implementar os quatro níveis de avaliação de treinamento de equipes: um guia prático. Rio de Janeiro: SENAC Rio.

Knowles. M. S.; Holton III, E. F.; Swanson, R. A. (2009) Aprendizagem de resultados - uma abordagem prática para aumentar a efetividade da educação corporativa. Rio de Janeiro: Elsevier.

Kolb, D. A. (1984) Experiential learning: experience as the source of learning and development. New Jersey, EUA: Prentice-Hall.

Lenci, A. (2010) The life cycle of knowledge. In Ontology and the lexicon, A natural language processing perspective. Redaktor Chu-Ren Huang.Cambridge; Cambridge University Press; 2010.Disponível em http://www.humnet.unipi.it/linguistica/ Docenti/Lenci/papers/ch14_cup-ontolex.pdf. Acesso em 20 de janeiro de 2014.

Lewis, M. A.; Maylor, H. R. (2007) Game playing and operations management education. Operations and Supply Group, Int. J. Production Economics 105 (2007) 134-149. DOI:10.1016/j.ijpe.2006.02.009. Disponível em www.elsevier.com/locate/ijpe.

Malhotra, N. K. (2001) Pesquisa de marketing: uma orientação aplicada. Porto Alegre: Bookman.

Martinelli, D. P. (1987) A utilização dos jogos de empresas no ensino de administração. Dissertação de mestrado. São Paulo: FEA/USP, 1987. 262 p.

Mattar, F. N. (2008) Pesquisa de Marketing: metodologia e planejamento. São Paulo: Atlas.
MEC (2010) MINISTÉRIO DA EDUCAÇÃO. Marco de ação de Belém. Sexta Conferência Internacional de Educação de Adultos. Brasília, 2010.

Minayo, M C. de S.(2012) O envelhecimento da população brasileira e os desafios para o setor de saúde. Cad. Saúde Pública, Rio de Janeiro, 28 (2):208-209, fev, 2012. Disponível em: www.scielosp.org/pdf/csp/v28n2/01.pdf . Acesso em: 28 de janeiro de 2014.

Miyashita, R.; Oliveira, L. F. V.S. de M.; Yoshizaki, H. T. Y. (2003) Os jogos de empresas como instrumento de treinamento em logística empresarial. In: Simpósio de Engenharia de Produção, 10, p. 1-10, 2003, Bauru.

Oliveira, M. A. (2009) Laboratório de gestão: implantando um modelo sistêmico de educação em administração. Tese de Doutorado. São Paulo: FEA/USP, 2009.

Pozo, H. (2004) Administração de recursos materiais e patrimoniais: uma abordagem logística. $3^{\mathrm{a}} \mathrm{Ed}$. São Paulo: Atlas.

Rogers, C. R. (1985) Liberdade de aprender em nossa década. Porto Alegre: Artes Médicas.

Sauaia, A. C. A. (2008) Laboratório de gestão: simulador organizacional, jogo de empresas e pesquisa aplicada. Barueri: Manole.

Serapicos, M. do A. L. (2008) Reconhecimento e validação da aprendizagem ao longo da vida. Dissertação de Mestrado. Universidade de Aveiro, 2008. Disponível em http://hdl.handle.net/10773/3373. Acesso em 22 de fevereiro de 2013.

Silva, E. L. da; Menezes, E. M. (2001) Metodologia da pesquisa $e$ elaboração de dissertação. ${ }^{\mathrm{a}}$ ed. UFSC/PPGEP/LED, Florianópolis, 2001.

Sitoe, R. M. (2006) Aprendizagem ao longo da vida: um conceito utópico?.Comport. Organ. Gest., Lisboa, v.12, n.2, 2006. Disponível em http://www.scielo.gpeari.mctes.pt/scielo.php?script=sci_arttext $\& \mathrm{pid}=$ S0872-96622006000200009\&lng=pt\&nrm=iso. Acesso em 10 abr. 2013.

Tanabe, M. (1977) Jogos de empresas. Dissertação de mestrado. São Paulo: FEA/USP, 1977. 117p. 